\title{
The Initial-Value Adjusting Method for Problems of the Least Squares Type of Ordinary Differential Equations
}

By

\author{
Taketomo Mitsui*
}

\section{Introduction}

This work is concerned with the numerical processes to solve problems of the least squares type for ordinary differential equations. Consider the differential equation

$$
\frac{d x}{d t}=X(t, x), \quad a<t<b
$$

where $x$ and $X(t, x)$ are real $n$-dimensional vectors and try to obtain a solution $x=x(t)$ of the above equation on the interval $[a, b]$ such that

$$
\frac{1}{2} \sum_{j=1}^{N}{ }^{t}\left[L_{j} x\left(t_{j}\right)-d_{j}\right]\left[L_{j} x\left(t_{j}\right)-d_{j}\right]=\text { minimum }
$$

where $t_{j}$ are given points on $[a, b], a=t_{1}<t_{2}<\cdots<t_{N}=b$, and $L_{j}$ and $d_{j}$ are given matrices and vectors, respectively.

Some authors have discussed these types of problems and proposed several numerical methods to solve them. Banks and Groome [1] have discussed the quasilinearization algorithm and established the quadratic convergence of it. Urabe [8] has shown that the problem can be reduced to the multipoint boundary value problem of nonlinear boundary condition, and proposed the application of the Newton iterative process. After Urabe's works, Fujii [2] has shown another reduction of the problem to a boundary value problem and discussed the Chebyshev-series-approximation with a-posteriori error bound.

In the present paper, we first propose a new method which is an applied version of the initial-value adjusting method given by Ojika and Kasue [6] for

Received January 29, 1979.

* Research Institute for Mathematical Sciences, Kyoto University, Kyoto 606, Japan. 
nonlinear boundary value problems of ordinary differential equations (on an analysis of the method, see also [5]). In the next sections, we analyse the process and prove the convergence of an approximate value to the exact one under some conditions, roughly speaking, of sufficient smoothness of $X$ and suitable choice of the initial data of the iteration. Our method would be expected to have the advantages that they are easy to understand and program the algorithm. Finally, in the last section, an illustrative numerical example will be given.

\section{$\S 1$. The Initial-Value Adjusting Algorithm}

At first, we shall state the problem clearly. Consider a nonlinear differential equation

$$
\frac{d x}{d t}=X(t, x), \quad a<t<b .
$$

Here $x$ and $X(t, x)$ are $n$-dimensional vectors. Find a solution $x(t)$ of (1.1) which minimizes the following value

$$
J=\frac{1}{2} \sum_{j=1}^{N}{ }^{t}\left[L_{j} x\left(t_{j}\right)-d_{j}\right]\left[L_{j} x\left(t_{j}\right)-d_{j}\right],
$$

where $t_{j}(j=1, \ldots, N)$ are the given points on the interval $I, a=t_{1}<t_{2}<\cdots<t_{N}$ $=b, L_{j}$ and $d_{j}(j=1, \ldots, N)$ are the given $n \times n$ matrices and $n$-dimensional vectors, respectively. As far as the equation (1.1) is stable, the problem is equivalent to find an initial value $\eta$ such that the solution $x(t)$ starting with $x(a)=\eta$ minimizes $J$.

Let us define a linear operator $\boldsymbol{L}: C(I) \rightarrow \boldsymbol{R}^{n N}$ such that for $x \in C(I)$

$$
\mathbf{L} x={ }^{t}\left(L_{1} x\left(t_{1}\right), \ldots, L_{N} x\left(t_{N}\right)\right)
$$

Denote the $n N$-dimensional vector ${ }^{t}\left(d_{1}, \ldots, d_{N}\right)$ by $\boldsymbol{d}$. Note that $J$ is represented by

$$
J=\frac{1}{2} t\{\boldsymbol{L} x-\boldsymbol{d}\}\{\boldsymbol{L} x-\boldsymbol{d}\}
$$

Our algorithm is expressed in the following steps.

Step 0 . Choose a suitable positive perturbation parameter $\varepsilon$ and an initial value $\eta_{0} \in \boldsymbol{R}^{n}$, and set $k=0$.

Step 1. Compute the numerical solution $x_{k}(t)$ of (1.1) with the initial con- 
dition $x_{k}(a)=\eta_{k}$, and obtain the resulting values $\mathbb{L}_{k}=\mathbb{L} x_{k}$ and $J_{k}=\frac{1}{2} t\left\{\boldsymbol{L}_{k}-\boldsymbol{d}\right\}$ $\times\left\{\boldsymbol{L}_{k}-\boldsymbol{d}\right\}$.

Step 2. If the value $J_{k}$ differs from $J_{k-1}$ by less than a specified value, terminate the iteration. Otherwise, go to the next step. (If $k=0$, skip this step.)

Step 3. Set $j=1$.

Step 4. Compute the numerical solution $y_{k}^{(j)}(t)$ of (1.1) with the initial condition $y_{k}^{(j)}(a)=\eta_{k}+\varepsilon e_{j}$. Here $e_{j}$ means the $j$-th unit vector of $\boldsymbol{R}^{n}$.

Step 5. Replace $j$ by $j+1$, and return to Step 4 until $j=n$.

Step 6. Determine the $n N \times n$ matrix $S\left(\varepsilon ; x_{k}\right)$ (the adjusting matrix) such that

$$
S\left(\varepsilon ; x_{k}\right)=\left(\frac{1}{\varepsilon}\left\{\boldsymbol{L} y_{k}^{(1)}-\boldsymbol{L}_{k}\right\}, \ldots, \frac{1}{\varepsilon}\left\{\boldsymbol{L} y_{k}^{(j)}-\mathbb{L}_{k}\right\}, \ldots, \frac{1}{\varepsilon}\left\{\mathbb{L} y_{k}^{(n)}-\mathbb{L}_{k}\right\}\right) .
$$

Step 7. Determine the initial value $\eta_{k+1}$ for the next iteration by

$$
\eta_{k+1}=\eta_{k}-\left\{{ }^{t} S\left(\varepsilon ; x_{k}\right) S\left(\varepsilon ; x_{k}\right)\right\}^{-1}{ }^{t} S\left(\varepsilon ; x_{k}\right)\left\{\mathbb{L}_{k}-\mathbb{d}\right\} .
$$

Then replace $k$ by $k+1$, and return to Step 1 .

Computational Remarks. In the above process, the numerical integration of the differential equation (1.1) are carried out by a suitable step-by-step method, for example, the Runge-Kutta method. Since the matrix ${ }^{t} S\left(\varepsilon ; x_{k}\right) S(\varepsilon$; $x_{k}$ ) is an $n \times n$ symmetric, positive definite matrix, the square-root-free Cholesky's method is preferable for the solution of the linear equations in (1.6).

In what follows we shall denote the Euclidean norm of an $n$-dimensional vector $x$ by $\|x\| . \quad C(I)$ stands for the Banach space of vector-valued continuous functions on $I$, equipped with the norm

$$
\|x\|_{C}=\max _{t \in I}\|x(t)\| .
$$

$C^{1}(I)$ means a subset of $C(I)$ of continuously differentiable functions on $I$. The norms for matrices $\boldsymbol{R}^{m} \rightarrow \boldsymbol{R}^{m^{\prime}}$ and other linear operators should be taken as the induced norms by the corresponding vector norms.

Let $\mathscr{D}$ be the domain of the $t x$-space bounded on $x$, intercepted by two hyperplanes $t=a$ and $t=b$. The boundary points of $\mathscr{D}$ on the hyperplanes $t=a$ and $t=b$ are supposed to be included in $\mathscr{D}$ and to make an open set on each hyperplane. Put

$$
\begin{array}{lll}
D=\left\{x \in C^{1}(I) ;(t, x(t)) \in \mathscr{D}\right. & \text { for } & t \in I\} \\
D^{\prime}=\{x \in C(I) ;(t, x(t)) \in \mathscr{D} & \text { for } & t \in I\}
\end{array}
$$


We shall need the following assumptions to the problem (1.1) and (1.2).

Assumption 1. (i) $X(t, x)$ is defined and three-times continuously differentiable with respect to $x$ on $\mathscr{D}$. $X$ and its derivatives up to the third order are continuous with respect to $t$ on $I$.

(ii) In $\mathscr{D}$, there exist a positive number $\delta_{0}$ and a mapping $Q(\xi ; \cdot): \boldsymbol{R}^{n} \rightarrow$ $\boldsymbol{R}^{n}$ such that the equation

$$
X(t, x+\xi)=X(t, x)+X_{x}(t, x) \xi+\frac{1}{2} X_{x x}(t, x) \xi \xi+Q(\xi ; x)
$$

holds whenever $\|\xi\| \leqq \delta_{0}$ for each $(t, x) \in \mathscr{D}$. Here $X_{x}(t, x)$ is the Jacobian matrix of $X(t, x)$ with respect to $x$ and $X_{x x}(t, x)$ stands for the tensor of the third order with components $\partial^{2} X_{j}(t, x) / \partial x_{k} \partial x_{l}(j, k, l=1,2, \ldots, n)$.

(iii) The constants $K_{0} \sim K_{3}$ can be defined finitely as following:

$$
\begin{aligned}
& K_{0}=\sup _{\mathscr{D}}\|X(t, x)\|, \\
& K_{1}=\sup _{\mathscr{D}}\left\|X_{x}(t, x)\right\|, \\
& K_{2}=\sup _{\mathscr{D}}\left\|X_{x x}(t, x)\right\|, \\
& \|Q(\xi ; x)\| \leqq K_{3}\|\xi\|^{3} \text { for }\|\xi\| \leqq \delta_{0} \text { uniformly to }(t, x) \in \mathscr{D} .
\end{aligned}
$$

(iv) For arbitrary scalar number $\lambda$, the equality $Q(\lambda \xi ; x)=\lambda^{3} Q(\xi ; x)$ holds. If $\xi_{1}, \xi_{2}, \xi_{3}$ are such vectors that $\left\|\xi_{1}+\xi_{2}\right\|$, $\left\|\xi_{1}\right\|,\left\|\xi_{3}\right\| \leqq \delta_{0}$, the equation

$$
\begin{aligned}
& Q\left(\xi_{1}+\xi_{2} ; x\right)-Q\left(\xi_{1} ; x\right)-Q\left(\xi_{3} ; x\right) \\
& \quad=\xi_{1} q_{1}\left(\xi_{2}\right)+\xi_{2} q_{2}\left(\xi_{1}\right)+\left(\xi_{2}-\xi_{3}\right) q_{3}\left(\xi_{2}, \xi_{3}\right)
\end{aligned}
$$

holds for $(t, x) \in \mathscr{D}$. Here $q_{1}(\xi)$ and $q_{2}(\xi)$ are of the behaviour of the quadratic form of $\xi$, i.e.

$$
\lim _{\|\xi\| \rightarrow 0} q_{1}(\xi) /\|\xi\|^{2}, \quad \lim _{\|\xi\| \rightarrow 0} q_{2}(\xi) /\|\xi\|^{2}<+\infty,
$$

and $q_{3}$ has the estimation

$$
\left\|q_{3}\left(\xi_{2}, \xi_{3}\right)\right\| \leqq K_{3}^{\prime}
$$

for $\left\|\xi_{2}\right\|,\left\|\xi_{3}\right\| \leqq \delta_{0}$.

Because of the equation (1.7), put

$$
X(t, x+\xi)=X(t, x)+X_{x}(t, x) \xi+R(\xi ; x),
$$

then it is evident that $R(\xi ; x)$ has the estimation

$$
\|R(\xi ; x)\| \leqq K_{2}^{\prime}\|\xi\|^{2}
$$


and is Lipschitz-continuous

$$
\left\|R\left(\xi_{1} ; x\right)-R\left(\xi_{2} ; x\right)\right\| \leqq L_{R}\left\|\xi_{1}-\xi_{2}\right\|
$$

for $\left\|\xi_{1}\right\|,\left\|\xi_{2}\right\| \leqq \delta_{0}$.

Let us consider an operator $\mathscr{F}$ mapping $\mathbb{R}^{n}$ to a function of $C^{1}(I)$ along the flow generated by the differential equation (1.1) such that

$$
\begin{aligned}
& \frac{d}{d t} \mathscr{F} \eta=X[t, \mathscr{F} \eta(t)], \quad a<t<b, \\
& \mathscr{F} \eta(a)=\eta .
\end{aligned}
$$

If $\eta^{*}$ is a vector such that $\left(a, \eta^{*}\right) \in \mathscr{D}$ and $\eta^{*}$ is a minimal solution for

$$
J(\eta) \equiv \frac{1}{2} t\{\mathbf{L F} \eta-\boldsymbol{d}\}\{\mathbb{L} \mathscr{F} \eta-\boldsymbol{d}\},
$$

then $\left.\operatorname{grad} J(\eta)\right|_{\eta=\eta^{*}}=0$ holds, that is

$$
{ }^{t}\left\{\boldsymbol{L} \Phi\left(\cdot ; \mathscr{F} \eta^{*}\right)\right\}\left\{\boldsymbol{L} \mathscr{F} \eta^{*}-\boldsymbol{d}\right\}=0,
$$

where $\Phi(t ; x)$ denotes the matrizant of the linear homogeneous matrix differential equation

$$
\frac{d \Phi}{d t}=X_{x}[t, x(t)] \Phi, \quad a<t<b .
$$

Moreover, considering that $J$ is a quadratic form of vector, we may take the following assumption.

Assumption 2. There exists a vector $\eta^{*}$ such that $\left(a, \eta^{*}\right) \in \mathscr{D}, \mathscr{F} \eta^{*} \in D$,

$$
{ }^{t}\left\{\boldsymbol{L} \Phi\left(\cdot ; \mathscr{F} \eta^{*}\right)\right\}\left\{\mathbf{L} \mathscr{F} \eta^{*}-\boldsymbol{d}\right\}=0
$$

and

$$
\operatorname{det}^{t}\left\{\mathbb{L} \Phi\left(\cdot ; \mathscr{F} \eta^{*}\right)\right\} \mathbb{L} \Phi\left(\cdot ; \mathscr{F} \eta^{*}\right) \neq 0
$$

hold.

Furthermore, there exists a positive constant $\bar{\varepsilon}$ such that for $0<\varepsilon \leqq \bar{\varepsilon}$ the initial value problem

$$
\begin{aligned}
& \frac{d y}{d t}=X(t, y), \quad a<t<b \\
& y(a)=\eta^{*}+\varepsilon e_{j}
\end{aligned}
$$

has a unique solution in $D$ for every $j$.

We shall call $\eta^{*}$ the exact (local) isolated minimal point of $J(\eta)$. 
Throughout the present paper we take the above two assumptions implicitly and will not mention in the statement of Theorems and Lemmas.

Then we shall make some preliminaries after [5].

The $j$-th column vector of $\Phi(t ; x)$, denoted by $\varphi^{(j)}(t)$, is bounded by

$$
\left\|\varphi^{(j)}\right\|_{C} \leqq e^{2 K_{1}(b-a)}
$$

for $x \in D^{\prime}$ and $j=1, \ldots, n$. Therefore the estimation

$$
\|\Phi(\cdot ; x)\| \leqq n e^{2 K_{1}(b-a)} \equiv M_{0}
$$

holds. We shall call the matrix $\boldsymbol{L} \Phi(\cdot ; \mathscr{F} \eta)$ the $G$-matrix and denote it by $G(\eta)$. Since $D$ is open in $C^{1}(I)$, for a positive constant $M_{1}$ we can take a positive number $\Delta$ such that in the $\Delta$-neighbourhood $B_{\Delta}$ of $\eta^{*}$ defined by

$$
B_{\Delta}=\left\{\eta \in \boldsymbol{R}^{n} ;\left\|\eta-\eta^{*}\right\| \leqq \Delta\right\},
$$

the inverse of compound $G$-matrix defined by $\left(\mathfrak{G}(\eta)={ }^{t} G(\eta) G(\eta)\right.$ exists and the estimation

$$
\left\|\mathfrak{G}(\eta)^{-1}\right\| \leqq M_{1}
$$

holds. Replace $\Delta$ by a suitable value less than $\Delta$ if necessary, then it is possible that the inequality

$$
\left\|\mathscr{F} \eta-\mathscr{F} \eta^{*}\right\|_{c} \leqq \delta_{0}
$$

holds for $\eta \in B_{\Delta}$. We shall fix the number $\Delta$.

Corresponding to our iterative process, an operator $\mathscr{A}$ mapping $\boldsymbol{R}^{n}$ into itself is defined by the following:

$$
\mathscr{A} \eta=\eta-\left\{{ }^{t} S(\varepsilon ; \mathscr{F} \eta) S(\varepsilon ; \mathscr{F} \eta)\right\}^{-1}{ }^{t} S(\varepsilon ; \mathscr{F} \eta)\{\boldsymbol{L F} \eta-d\} \text { for } \eta \in B_{\Delta},
$$

where the domain of $\mathscr{A}$ is identical to $B_{\Delta}$. Then the iterative process (1.6) is simply represented by

$$
\eta_{k+1}=\mathscr{A} \eta_{k}, \quad k=0,1,2, \ldots
$$

Lemmas $2 \sim 4$ in [5] assert the followings.

For $\eta \in B_{\Delta}$, put $x(t)=\mathscr{F} \eta(t) . y^{(j)}(t)$ in Step 4 of the algorithm has the following expression.

$$
y^{(j)}(t)=x(t)+\varepsilon\left\{\varphi^{(j)}(t)+v^{(j)}(t)\right\} \quad \text { on } \quad I,
$$

where $v^{(j)}(t)$ satisfies the differential equation 


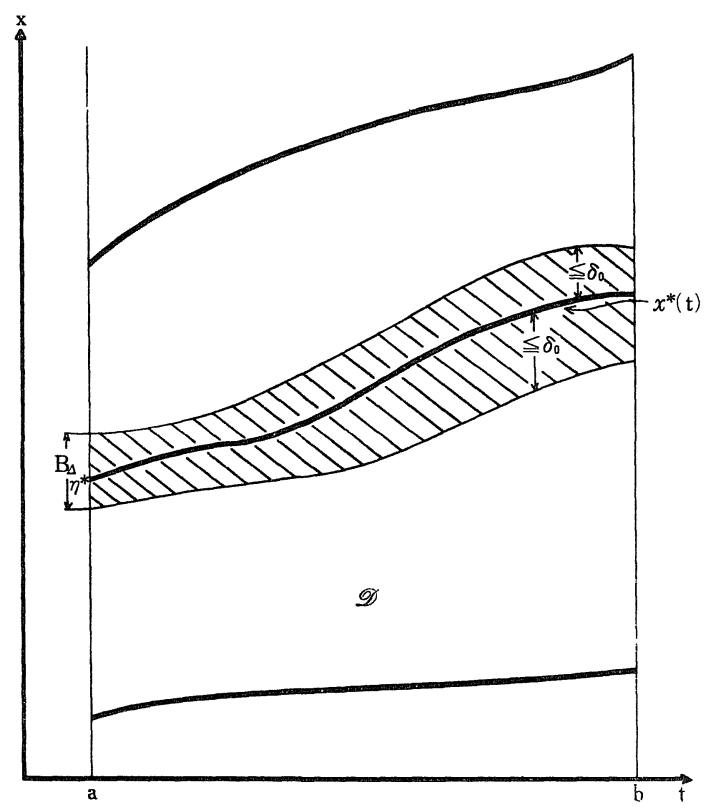

Figure 1.1.

$$
\begin{aligned}
\frac{d v^{(j)}(t)}{d t}= & \frac{1}{\varepsilon}\left\{X\left[t, x(t)+\varepsilon\left\{\varphi^{(j)}(t)+v^{(j)}(t)\right\}\right]-X[t, x(t)]\right\} \\
& -X_{x}[t, x(t)] \varphi^{(j)}(t), \quad a<t<b
\end{aligned}
$$

with the initial condition

$$
v^{(j)}(a)=0 .
$$

For arbitrary small positive number $\varepsilon$ there exists a positive constant $C^{*}$ such that $v^{(j)}(t)$ satisfies

$$
\left\|v^{(j)}\right\|_{C} \leqq C^{*} \varepsilon .
$$

There exists a small positive number $\varepsilon_{0}$ such that for $0 \leqq \varepsilon \leqq \varepsilon_{0}$ the estimation

$$
\|S(\varepsilon ; \mathscr{F} \eta)-G(\eta)\| \leqq C^{* *} \varepsilon \equiv \delta_{1}
$$

holds in $B_{\Delta}$. Moreover for $0 \leqq \varepsilon \leqq \varepsilon_{0}$ and $\eta \in B_{\Delta}$ the inverse of the compound adjusting matrix ${ }^{t} S(\varepsilon ; \mathscr{F} \eta) S(\varepsilon ; \mathscr{F} \eta)$ exists and the estimation

$$
\left\|\left\{{ }^{t} S(\varepsilon ; \mathscr{F} \eta) S(\varepsilon ; \mathscr{F} \eta)\right\}^{-1}\right\| \leqq \frac{M_{1}}{1-\delta_{1} M_{1}\left(2 L_{0} M_{0}+\delta_{1}\right)}
$$

holds, where $L_{0}$ is the bound of the operator norm of $\boldsymbol{L}$ on $D$. 


\section{§2. Fixed Point of the Operator}

It is noteworthy that the exact minimal point $\eta^{*}$ is not a fixed point of $\mathscr{A}$, because a fixed point of $\mathscr{A}$ must satisfy the equation

$$
\left\{{ }^{t} S(\varepsilon ; \mathscr{F} \eta) S(\varepsilon ; \mathscr{F} \eta)\right\}^{-1}{ }^{t} S(\varepsilon ; \mathscr{F} \eta)\{\boldsymbol{L} \mathscr{F} \eta-d\}=0,
$$

i.e.

$$
{ }^{t} S(\varepsilon ; \mathscr{F} \eta)\{\boldsymbol{L} \mathscr{F} \eta-\boldsymbol{d}\}=0 .
$$

On the other hand, from (1.13) $\eta^{*}$ satisfies

$$
{ }^{t} G\left(\eta^{*}\right)\left\{\boldsymbol{L} \mathscr{F} \eta^{*}-\boldsymbol{d}\right\}=0 .
$$

$S(\varepsilon ; \mathscr{F} \eta)$ is surely an approximation of $G(\eta)$, but is not identical to that as far as $\varepsilon \neq 0$.

Therefore, we should answer the question whether a fixed point of $\mathscr{A}$ exists. Throughout this section we shall allow a negative value for $\varepsilon$ for convenience' sake. In the case of negative $\varepsilon$, we can define the matrix $S(\varepsilon ; \mathscr{F} \eta)$ and the operator $\mathscr{A}$ in the natural way. The estimations (1.23) (1.25) hold by replacing $\varepsilon$ by $|\varepsilon|$.

We shall prove the existence of the fixed point of $\mathscr{A}$ in some neighbourhood of $\eta^{*}$ by the implicit function theorem.

Define a function $\mathscr{T}(\eta, \varepsilon)$ by

$$
\mathscr{T}(\eta, \varepsilon)={ }^{t} S(\varepsilon ; \mathscr{F} \eta)\{\boldsymbol{L} \mathscr{F} \eta-\boldsymbol{d}\}
$$

for $(\eta, \varepsilon)$ such that $\eta \in B_{\Delta}$ and $0<|\varepsilon| \leqq \varepsilon_{0}$.

Lemma 1. Define $\mathscr{T}(\eta, 0)$ by

$$
\mathscr{T}(\eta, 0)=\lim _{\varepsilon \rightarrow 0} \mathscr{T}(\eta, \varepsilon)
$$

then

$$
\mathscr{T}(\eta, 0)={ }^{t} G(\eta)\{\boldsymbol{L} \mathscr{F} \eta-\boldsymbol{d}\}
$$

holds.

Proof is evident.

Lemma 2. For $\eta \in B_{\Delta}$ and $\left|\varepsilon_{1}\right|,\left|\varepsilon_{2}\right| \leqq \varepsilon_{0}$, the estimation

$$
\left\|S\left(\varepsilon_{1} ; \mathscr{F} \eta\right)-S\left(\varepsilon_{2} ; \mathscr{F} \eta\right)\right\| \leqq \text { const. }\left|\varepsilon_{1}-\varepsilon_{2}\right|
$$


holds. Here the constant does not depend on $\eta, \varepsilon_{1}$ and $\varepsilon_{2}$.

Proof. Let us denote the $j$-th column vector of $S(\varepsilon ; \mathscr{F} \eta)$ by $s_{j}(\varepsilon ; \mathscr{F} \eta)$. Put

$$
\mathscr{F}\left(\eta+\varepsilon_{1} e_{j}\right)=y_{1}^{(j)}=x+\varepsilon_{1}\left\{\varphi^{(j)}+v_{1}^{(j)}\right\}
$$

and

$$
\mathscr{F}\left(\eta+\varepsilon_{2} e_{j}\right)=y_{2}^{(j)}=x+\varepsilon_{2}\left\{\varphi^{(j)}+v_{2}^{(j)}\right\}
$$

(see (1.20)), then the equation

$$
s_{j}\left(\varepsilon_{1} ; \mathscr{F} \eta\right)-s_{j}\left(\varepsilon_{2} ; \mathscr{F} \eta\right)=\mathbb{L}\left(v_{1}^{(j)}-v_{2}^{(j)}\right)
$$

holds. Define $w^{(j)}$ by $w^{(j)}=v_{1}^{(j)}-v_{2}^{(j)}$, then $w^{(j)}$ must satisfy the differential equation

$$
\begin{aligned}
\frac{d w^{(j)}}{d t}= & \frac{1}{\varepsilon_{1}}\left\{X\left[t, y_{1}^{(j)}(t)\right]-X[t, x(t)]\right\} \\
& -\frac{1}{\varepsilon_{2}}\left\{X\left[t, y_{2}^{(j)}(t)\right]-X[t, x(t)]\right\}
\end{aligned}
$$

with the initial condition

$$
w^{(j)}(a)=0 .
$$

By virtue of Assumption 1 and the equation (1.10),

(the right-hand side of (2.6))

$$
\begin{aligned}
= & \frac{1}{\varepsilon_{1}}\left\{X_{x}[t, x(t)] \varepsilon_{1}\left\{\varphi^{(j)}(t)+v_{1}^{(j)}(t)\right\}+R\left[y_{1}^{(j)}-x ; x\right]\right\} \\
& -\frac{1}{\varepsilon_{2}}\left\{X_{x}[t, x(t)] \varepsilon_{2}\left\{\varphi^{(j)}(t)+v_{2}^{(j)}(t)\right\}+R\left[y_{2}^{(j)}-x ; x\right]\right\} \\
= & X_{x}[t, x(t)] w^{(j)}(t)+\frac{1}{\varepsilon_{1}} R\left[y_{1}^{(j)}-x ; x\right]-\frac{1}{\varepsilon_{2}} R\left[y_{2}^{(j)}-x ; x\right] \\
= & X_{x}[t, x(t)] w^{(j)}(t)+\varepsilon_{1}\left\{R\left[\varphi^{(j)}+v_{1}^{(j)} ; x\right]-R\left[\varphi^{(j)}+v_{2}^{(j)} ; x\right]\right\} \\
& +\left(\varepsilon_{1}-\varepsilon_{2}\right) R\left[\varphi^{(j)}+v_{2}^{(j)} ; x\right] .
\end{aligned}
$$

Therefore, the integral form

$$
\begin{aligned}
w^{(j)}(t)= & \int_{a}^{t} X_{x}[s, x(s)] w^{(j)}(s) d s \\
& +\varepsilon_{1} \int_{a}^{t}\left\{R\left[\varphi^{(j)}(s)+v_{1}^{(j)}(s) ; x\right]-R\left[\varphi^{(j)}(s)+v_{2}^{(j)}(s) ; x\right]\right\} d s \\
& +\left(\varepsilon_{1}-\varepsilon_{2}\right) \int_{a}^{t} R\left[\varphi^{(j)}(s)+v_{2}^{(j)}(s) ; x\right] d s
\end{aligned}
$$

holds, which brings the inequality 
(2.9) $\left\|w^{(j)}(t)\right\| \leqq K_{1} \int_{a}^{t}\left\|w^{(j)}(s)\right\| d s$

$$
\begin{aligned}
& +\left|\varepsilon_{1}\right| \int_{a}^{t}\left\|R\left[\varphi^{(j)}(s)+v_{1}^{(j)}(s) ; x\right]-R\left[\varphi^{(j)}(s)+v_{2}^{(j)}(s) ; x\right]\right\| d s \\
& +\left|\varepsilon_{1}-\varepsilon_{2}\right| \int_{a}^{t}\left\|R\left[\varphi^{(j)}(s)+v_{2}^{(j)}(s) ; x\right]\right\| d s .
\end{aligned}
$$

Because of the estimations (1.11) and (1.12) we have

$$
\begin{aligned}
\left\|w^{(j)}(t)\right\| \leqq & K_{1} \int_{a}^{t}\left\|w^{(j)}(s)\right\| d s+\left|\varepsilon_{1}\right| L_{R} \int_{a}^{t}\left\|w^{(j)}(s)\right\| d s \\
& +\left|\varepsilon_{1}-\varepsilon_{2}\right| K_{2}^{\prime} \delta_{0}^{2}(b-a) \\
\leqq & \left|\varepsilon_{1}-\varepsilon_{2}\right| K_{2}^{\prime} \delta_{0}^{2}(b-a)+\left(K_{1}+\varepsilon_{0} L_{R}\right) \int_{a}^{t}\left\|w^{(j)}(s)\right\| d s
\end{aligned}
$$

Applying the Gronwall's lemma to (2.10), it holds that

$$
\left\|w^{(j)}(t)\right\| \leqq\left|\varepsilon_{1}-\varepsilon_{2}\right| K_{2}^{\prime} \delta_{0}^{2}(b-a) e^{\left(K_{1}+\varepsilon_{0} L_{R}\right)(t-a)},
$$

which implies

$$
\left\|w^{(j)}\right\|_{C} \leqq\left|\varepsilon_{1}-\varepsilon_{2}\right| K_{2}^{\prime} \delta_{0}^{2}(b-a) e^{\left(K_{1}+\varepsilon_{0} L_{R}\right)(b-a)}
$$

Lemma 3. For sufficiently small positive numbers $\tilde{\Delta}$ and $\tilde{\varepsilon}$, the relation

$$
S(\varepsilon ; \mathscr{F}(\eta+\xi))-S(\varepsilon ; \mathscr{F} \eta)=\boldsymbol{L} P(\cdot ; \mathscr{F} \eta, \varepsilon) \xi+o(\|\xi\|)
$$

holds if $\eta, \eta+\xi \in B_{\tilde{\Delta}}$ and $|\varepsilon| \leqq \tilde{\varepsilon}$, where $P(t ; \mathscr{F} \eta, \varepsilon)$ is a linear mapping which maps $\xi \in \boldsymbol{R}^{n}$ to an $n \times n$ matrix $P(t ; \mathscr{F} \eta, \varepsilon) \xi$ with components of continuous functions on $I$.

Proof. We shall put

$$
\begin{aligned}
& \mathscr{F}(\eta+\xi)=x_{1}, \quad \mathscr{F} \eta=x_{2}, \quad \mathscr{F}\left(\eta+\xi+\varepsilon e_{j}\right)=y_{1}^{(j)}=x_{1}+\varepsilon u_{1}^{(j)}, \\
& \mathscr{F}\left(\eta+\varepsilon e_{j}\right)=y_{2}^{(j)}=x_{2}+\varepsilon u_{2}^{(j)} .
\end{aligned}
$$

Then we have

$$
\begin{aligned}
s_{j}(\varepsilon ; \mathscr{F}(\eta+\xi))-s_{j}(\varepsilon ; \mathscr{F} \eta) & =\frac{1}{\varepsilon}\left\{\boldsymbol{L} y_{1}^{(j)}-\boldsymbol{L} x_{1}-\boldsymbol{L} y_{2}^{(j)}+\boldsymbol{L} x_{2}\right\} \\
& =\boldsymbol{L}\left(u_{1}^{(j)}-u_{2}^{(j)}\right)
\end{aligned}
$$

Define $w^{(j)}$ by $w^{(j)}=u_{1}^{(j)}-u_{2}^{(j)}$, then $w^{(j)}$ must satisfy the differential equation

$$
\begin{aligned}
\frac{d w^{(j)}}{d t}= & \frac{1}{\varepsilon}\left\{X\left[t, x_{1}(t)+\varepsilon u_{1}^{(j)}(t)\right]\right. \\
& \left.-X\left[t, x_{1}(t)\right]-X\left[t, x_{2}(t)+\varepsilon u_{2}^{(j)}(t)\right]+X\left[t, x_{2}(t)\right]\right\}, \quad a<t<b,
\end{aligned}
$$

with the initial condition 


$$
w^{(j)}(a)=0 \text {. }
$$

Because of the equation (1.7), the right-hand side of (2.14) can be written as following:

$$
\begin{aligned}
\frac{1}{\varepsilon}\{ & X\left[t, x_{2}(t)\right]+X_{x}\left[t, x_{2}(t)\right]\left(x_{1}-x_{2}+\varepsilon u_{1}^{(j)}\right) \\
& +\frac{1}{2} X_{x x}\left[t, x_{2}(t)\right]\left(x_{1}-x_{2}+\varepsilon u_{1}^{(j)}\right)\left(x_{1}-x_{2}+\varepsilon u_{1}^{(j)}\right)+Q\left(x_{1}-x_{2}+\varepsilon u_{1}^{(j)} ; x_{2}\right) \\
& -X\left[t, x_{2}(t)\right]-X_{x}\left[t, x_{2}(t)\right]\left(x_{1}-x_{2}\right)-\frac{1}{2} X_{x x}\left[t, x_{2}(t)\right]\left(x_{1}-x_{2}\right)\left(x_{1}-x_{2}\right) \\
& -Q\left(x_{1}-x_{2} ; x_{2}\right) \\
& -X\left[t, x_{2}(t)\right]-X_{x}\left[t, x_{2}(t)\right] \varepsilon u_{2}^{(j)}-\frac{1}{2} X_{x x}\left[t, x_{2}(t)\right] \varepsilon u_{2}^{(j)} \varepsilon u_{2}^{(j)} \\
& \left.-Q\left(\varepsilon u_{2}^{(j)} ; x_{2}\right)+X\left[t, x_{2}(t)\right]\right\} \\
= & X_{x}\left[t, x_{2}(t)\right] w^{(j)}+\frac{1}{2} \varepsilon X_{x x}\left[t, x_{2}(t)\right]\left(u_{1}^{(j)}+u_{2}^{(j)}\right) w^{(j)} \\
& +X_{x x}\left[t, x_{2}(t)\right]\left(x_{1}-x_{2}\right) u_{1}^{(j)} \\
& +\frac{1}{\varepsilon}\left\{Q\left(x_{1}-x_{2}+\varepsilon u_{1}^{(j)} ; x_{2}\right)-Q\left(x_{1}-x_{2} ; x_{2}\right)-Q\left(\varepsilon u_{2}^{(j)} ; x_{2}\right)\right\} \\
= & X_{x}\left[t, x_{2}(t)\right] w^{(j)}+\varepsilon X_{x x}\left[t, x_{2}(t)\right] u_{2}^{(j)} w^{(j)}+X_{x x}\left[t, x_{2}(t)\right]\left(x_{1}-x_{2}\right) u_{2}^{(j)} \\
& +X_{x x}\left[t, x_{2}(t)\right]\left(x_{1}-x_{2}\right) w^{(j)}+\frac{1}{2} \varepsilon X_{x x}\left[t, x_{2}(t)\right] w^{(j)} w^{(j)} \\
& +\frac{1}{\varepsilon}\left\{Q\left(x_{1}-x_{2}+\varepsilon u_{1}^{(j)} ; x_{2}\right)-Q\left(x_{1}-x_{2} ; x_{2}\right)-Q\left(\varepsilon u_{2}^{(j)} ; x_{2}\right)\right\}
\end{aligned}
$$

Utilizing (1.8) and (1.9), we have

$$
\begin{aligned}
\frac{1}{\varepsilon} & \left\{Q\left(x_{1}-x_{2}+\varepsilon u_{1}^{(j)} ; x_{2}\right)-Q\left(x_{1}-x_{2} ; x_{2}\right)-Q\left(\varepsilon u_{2}^{(j)} ; x_{2}\right)\right\} \\
& =\varepsilon\left(x_{1}-x_{2}\right) q_{1}\left(u_{2}^{(j)}\right)+u_{1}^{(j)} q_{2}\left(x_{1}-x_{2}\right)+\varepsilon^{2} w^{(j)} q_{3}\left(u_{1}^{(j)}, u_{2}^{(j)}\right),
\end{aligned}
$$

which brings the equation

$$
\begin{aligned}
\frac{d w^{(j)}}{d t}= & \left\{X_{x}\left[t, x_{2}(t)\right]+\varepsilon X_{x x}\left[t, x_{2}(t)\right] u_{2}^{(j)}(t)\right\} w^{(j)} \\
& +X_{x x}\left[t, x_{2}(t)\right]\left(x_{1}(t)-x_{2}(t)\right) u_{2}^{(j)}(t) \\
& +X_{x x}\left[t, x_{2}(t)\right]\left\{x_{1}(t)-x_{2}(t)+\frac{1}{2} \varepsilon w^{(j)}\right\} w^{(j)} \\
& +u_{1}^{(j)}(t) q\left(x_{1}-x_{2}\right)+\varepsilon\left(x_{1}-x_{2}\right) q_{1}\left(u_{2}^{(j)}\right)+\varepsilon^{2} w^{(j)} q_{3}\left(u_{1}^{(j)}, u_{2}^{(j)}\right) .
\end{aligned}
$$

Let us denote the matrizant which corresponds to

$$
X_{x}\left[t, x_{2}(t)\right]+\varepsilon X_{x x}\left[t, x_{2}(t)\right] u_{2}^{(j)}
$$


by $\Psi_{j}(t ; \mathscr{F} \eta, \varepsilon)$ (note that $\Psi_{j}$ does not depend on $\xi$ ) and define a mapping $H_{j, \varepsilon}$ which maps a function $y(t) \in C(I)$ into $C^{1}(I)$ by

$$
\left[H_{j, \varepsilon} y\right](t)=\Psi_{j}(t ; \mathscr{F} \eta, \varepsilon) \int_{a}^{t} \Psi_{j}^{-1}(s ; \mathscr{F} \eta, \varepsilon) y(s) d s,
$$

then we can transform (2.16) and (2.15) into the integral equation

$$
\begin{aligned}
w^{(j)}(t)= & {\left[H_{j, \varepsilon} Y\{\mathscr{F}(\eta+\xi)-\mathscr{F} \eta\} u_{2}^{(j)}\right](t) } \\
& +\varepsilon\left[H_{j, \varepsilon}\{\mathscr{F}(\eta+\xi)-\mathscr{F} \eta\} q_{1}\left(u_{2}^{(j)}\right)\right](t) \\
& +\varepsilon^{2}\left[H_{j, \varepsilon}\left\{w^{(j)} q_{3}\left(u_{1}^{(j)}, u_{2}^{(j)}\right)\right\}\right](t) \\
& +\left[H_{j, \varepsilon} Y\left\{\mathscr{F}(\eta+\xi)-\mathscr{F} \eta+\frac{1}{2} \varepsilon w^{(j)}\right\} w^{(j)}\right](t) \\
& +\left[H_{j, \varepsilon}\left\{u_{1}^{(j)} q_{2}(\mathscr{F}(\eta+\xi)-\mathscr{F} \eta)\right\}\right](t) .
\end{aligned}
$$

Here $Y(t)$ stands for $X_{x x}[t, \mathscr{F} \eta(t)]$.

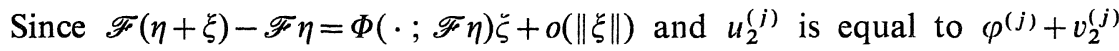
which is bounded, the first term of the right-hand side of (2.17) is the order $O(\|\xi\|)$. Also, the second term is the order $O(\|\xi\|)$. Moreover, because of (1.9) in Assumption 1, the last term is the order $o(\|\xi\|)$. There is no factor which brings terms of the order lower than $O(\|\xi\|)$ in the other terms of the right-hand side of (2.17), thus we can assert that $w^{(j)}(t)$ is $O(\|\xi\|)$ as a whole.

Define an $n \times n$ matrix $h_{1}(t)$ with continuous components by

$$
\left[H_{j, \varepsilon}\{\mathscr{F}(\eta+\xi)-\mathscr{F} \eta\} q_{1}\left(u_{2}^{(j)}\right)\right](t)=h_{1}^{(j)}(t) \xi+o(\|\xi\|) .
$$

In the same manner we have

$$
\left[H_{j, \varepsilon}\left\{w^{(j)} q_{3}\left(u_{1}^{(j)}, u_{2}^{(j)}\right)\right\}\right](t)=h_{2}^{(j)}(t) \xi+o(\|\xi\|) .
$$

where $h_{2}(t)$ is an $n \times n$ matrix with continuous components. Put

$$
w_{0}^{(j)}(t)=\left[H_{j, \varepsilon} Y\left\{\mathscr{F}(\eta+\xi)-\mathscr{F} \eta+\frac{1}{2} \varepsilon w^{(j)}\right\} w^{(j)}\right](t),
$$

then $H_{j, \varepsilon} Y\{\mathscr{F}(\eta+\xi)-\mathscr{F} \eta\} w^{(j)}$ is the order $O\left(\|\xi\|^{2}\right)$. On the other hand $\frac{1}{2} \varepsilon H_{j, \varepsilon} Y w^{(j)} W^{(j)}$ must be the order $O\left(\|\xi\|^{2}\right)$. Therefore $w_{0}^{(j)}=o(\|\xi\|)$ holds. Summing up the above considerations, the equation (2.17) can be rewritten as

$$
w^{(j)}(t)=\left[H_{j, \varepsilon} Y \Phi(\cdot ; \mathscr{F} \eta) \xi u_{2}^{(j)}\right](t)+\varepsilon h_{1}^{(j)}(t) \xi+\varepsilon^{2} h_{2}^{(j)}(t) \xi+o(\|\xi\|) .
$$

Hence, define the linear operator $P(t ; \mathscr{F} \eta, \varepsilon)$ such that $P(t ; \mathscr{F} \eta, \varepsilon) \xi$ is formed by

$$
\left[H_{j, \varepsilon} Y \Phi(\cdot ; \mathscr{F} \eta) \xi u_{2}^{(j)}\right](t)+\varepsilon h_{1}^{(j)}(t) \xi+\varepsilon^{2} h_{2}^{(j)}(t) \xi
$$


as the $j$-th column vector, then we have the conclusion.

Corollary. For sufficiently small positive numbers $\tilde{\Delta}$ and $\tilde{\varepsilon}$, the estimation

$$
\left\|S\left(\varepsilon ; \mathscr{F} \eta_{1}\right)-S\left(\varepsilon ; \mathscr{F} \eta_{2}\right)\right\| \leqq \text { const. }\left\|\eta_{1}-\eta_{2}\right\|
$$

holds if $\left\|\eta_{1}-\eta^{*}\right\|,\left\|\eta_{2}-\eta^{*}\right\| \leqq \tilde{\Delta}$ and $|\varepsilon| \leqq \tilde{\varepsilon}$. Here the constant does not depend on $\eta_{1}, \eta_{2}$ and $\varepsilon$.

Now we are ready to state

Theorem 1. There exist positive numbers $\Delta_{1}$ and $\varepsilon_{1}$ such that, for any $\varepsilon$ satisfying $|\varepsilon| \leqq \varepsilon_{1}$, the equation

$$
\mathscr{T}(\eta, \varepsilon)=0
$$

has a unique solution $\hat{\eta}=\hat{\eta}(\varepsilon)$ in the ball $\left\|\eta-\eta^{*}\right\| \leqq \Delta_{1}$, and $\hat{\eta} \rightarrow \eta^{*}$ as $\varepsilon \rightarrow 0$

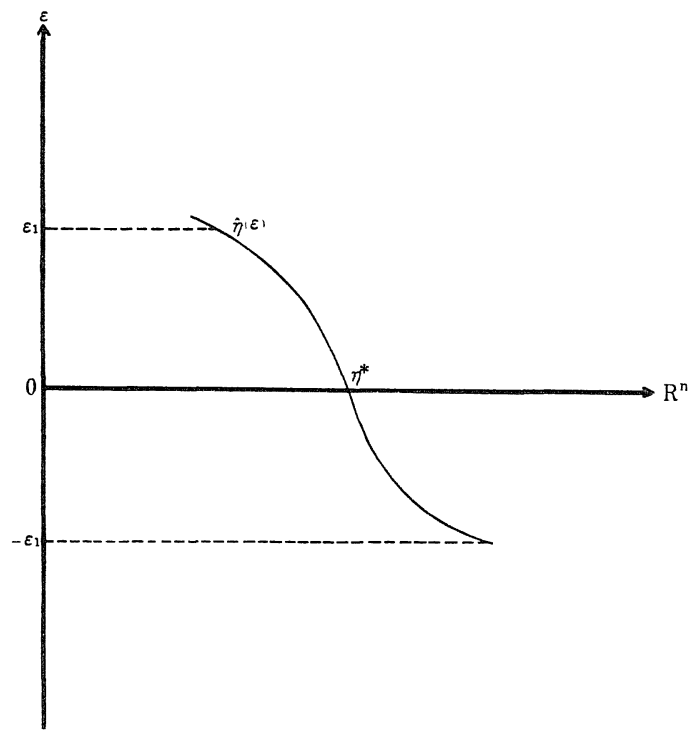

Figure 2.1.

Proof. We divide the proof into three parts.

First stage. Put the neighbourhood $N b$ of $\left(\eta^{*}, 0\right)$ as

$$
N b=\left\{(\eta, \varepsilon) ;\left\|\eta-\eta^{*}\right\| \leqq \tilde{\Delta},|\varepsilon| \leqq \tilde{\varepsilon}\right\},
$$

and assume that $N b$ is included in $B_{\Delta} \times\{\varepsilon ;|\varepsilon| \leqq \tilde{\varepsilon}\}$. For $\left(\eta_{1}, \varepsilon_{1}\right),\left(\eta_{2}, \varepsilon_{2}\right)$ in $N b$, the difference of $\mathscr{T}\left(\eta_{1}, \varepsilon_{1}\right)$ and $\mathscr{T}\left(\eta_{2}, \varepsilon_{2}\right)$ is written by 
(2.20)

$$
\begin{aligned}
\mathscr{T} & \left(\eta_{1}, \varepsilon_{1}\right)-\mathscr{T}\left(\eta_{2}, \varepsilon_{2}\right) \\
= & { }^{t} S\left(\varepsilon_{1} ; \mathscr{F} \eta_{1}\right)\left\{\boldsymbol{L} \mathscr{F} \eta_{1}-\boldsymbol{d}\right\}-{ }^{t} S\left(\varepsilon_{2} ; \mathscr{F} \eta_{2}\right)\left\{\boldsymbol{L} \mathscr{F} \eta_{2}-\boldsymbol{d}\right\} \\
= & { }^{t}\left\{S\left(\varepsilon_{1} ; \mathscr{F} \eta_{1}\right)-S\left(\varepsilon_{2} ; \mathscr{F} \eta_{1}\right)\right\}\left\{\boldsymbol{L} \mathscr{F} \eta_{1}-\boldsymbol{d}\right\} \\
& +{ }^{t}\left\{S\left(\varepsilon_{2} ; \mathscr{F} \eta_{1}\right)-S\left(\varepsilon_{2} ; \mathscr{F} \eta_{2}\right)\right\}\left\{\boldsymbol{L} \mathscr{F} \eta_{1}-\boldsymbol{d}\right\} \\
& +{ }^{t} S\left(\varepsilon_{2} ; \mathscr{F} \eta_{2}\right) \boldsymbol{L}\left(\mathscr{F} \eta_{1}-\mathscr{F} \eta_{2}\right) .
\end{aligned}
$$

Because of Lemma 2, the first term of the right-hand side of (2.20) is estimated by const. $\left|\varepsilon_{1}-\varepsilon_{2}\right|$. Besides, Corollary of Lemma 3 gives us the estimation

$$
\left\|^{t}\left\{S\left(\varepsilon_{2} ; \mathscr{F} \eta_{1}\right)-S\left(\varepsilon_{2} ; \mathscr{F} \eta_{2}\right)\right\}\left\{\boldsymbol{L} \mathscr{F} \eta_{1}-\boldsymbol{d}\right\}\right\| \leqq \text { const. }\left\|\eta_{1}-\eta_{2}\right\| .
$$

The inequality

$$
\left\|\mathscr{F} \eta_{1}-\mathscr{F} \eta_{2}\right\| \leqq \text { const. }\left\|\eta_{1}-\eta_{2}\right\|
$$

is easy to show.

Hence we have

$$
\left\|\mathscr{T}\left(\eta_{1}, \varepsilon_{1}\right)-\mathscr{T}\left(\eta_{2}, \varepsilon_{2}\right)\right\| \leqq \text { const. }\left|\varepsilon_{1}-\varepsilon_{2}\right|+\text { const. }\left\|\eta_{1}-\eta_{2}\right\| .
$$

This shows the joint continuity of $\mathscr{T}(\eta, \varepsilon)$ with respect to $\eta$ and $\varepsilon$.

Second stage. We shall calculate the Fréchet derivative of $\mathscr{T}(\eta, \varepsilon)$ with respect to $\eta$ in $N b$. If $\varepsilon \neq 0$, the equation

$$
\begin{aligned}
\mathscr{T} & (\eta+\xi, \varepsilon)-\mathscr{T}(\eta, \varepsilon) \\
= & { }^{t} S(\varepsilon ; \mathscr{F}(\eta+\xi))\{\boldsymbol{L} \mathscr{F}(\eta+\xi)-\boldsymbol{d}\}-{ }^{t} S(\varepsilon ; \mathscr{F} \eta)\{\boldsymbol{L} \mathscr{F} \eta-\boldsymbol{d}\} \\
= & { }^{t}\{S(\varepsilon ; \mathscr{F}(\eta+\xi))-S(\varepsilon ; \mathscr{F} \eta)\}\{\boldsymbol{L} \mathscr{F}(\eta+\xi)-\boldsymbol{d}\} \\
& +{ }^{t} S(\varepsilon ; \mathscr{F} \eta) \boldsymbol{L}(\mathscr{F}(\eta+\xi)-\mathscr{F} \eta)
\end{aligned}
$$

holds. By virtue of Lemma 3, we have

$$
\begin{gathered}
t\{S(\varepsilon ; \mathscr{F}(\eta+\xi))-S(\varepsilon ; \mathscr{F} \eta)\}\{\boldsymbol{L} \mathscr{F}(\eta+\xi)-\boldsymbol{d}\} \\
\quad={ }^{t}(\boldsymbol{L} P(\cdot ; \mathscr{F} \eta, \varepsilon) \xi)\{\boldsymbol{L} \mathscr{F} \eta-\boldsymbol{d}\}+o(\|\xi\|) .
\end{gathered}
$$

On the other hand,

$$
{ }^{t} S(\varepsilon ; \mathscr{F} \eta) \boldsymbol{L}(\mathscr{F}(\eta+\xi)-\mathscr{F} \eta)={ }^{t} S(\varepsilon ; \mathscr{F} \eta) G(\eta) \xi+o(\|\xi\|)
$$

holds. Therefore, from (2.21) we have

$$
\begin{aligned}
& \mathscr{T}(\eta+\xi, \varepsilon)-\mathscr{T}(\eta, \varepsilon) \\
& \quad={ }^{t}(\boldsymbol{L} P(\cdot ; \mathscr{F} \eta, \varepsilon) \xi)\{\boldsymbol{L} \mathscr{F} \eta-\boldsymbol{d}\}+{ }^{t} S(\varepsilon ; \mathscr{F} \eta) G(\eta) \xi+o(\|\xi\|),
\end{aligned}
$$

which implies that the Fréchet derivative $\mathscr{T}_{\eta}(\eta, \varepsilon)$ exists in $N b$ and is given by

$$
\mathscr{T}_{\eta}(\eta, \varepsilon) \xi={ }^{t}(\boldsymbol{L} P(\cdot ; \mathscr{F} \eta, \varepsilon) \dot{\zeta})\{\boldsymbol{L} \mathscr{F} \eta-\boldsymbol{d}\}+{ }^{t} S(\varepsilon ; \mathscr{F} \eta) G(\eta) \dot{\xi} .
$$


In the case $\varepsilon=0$, we obtain

$$
\begin{aligned}
\mathscr{T} & (\eta+\xi, 0)-\mathscr{T}(\eta, 0) \\
\quad & ={ }^{t} G(\eta+\xi)\{\boldsymbol{L} \mathscr{F}(\eta+\xi)-\boldsymbol{d}\}-{ }^{t} G(\eta)\{\mathbb{L} \mathscr{F} \eta-\boldsymbol{d}\} \\
& ={ }^{t}\{G(\eta+\xi)-G(\eta)\}\{\mathbb{L} \mathscr{F}(\eta+\xi)-\boldsymbol{d}\}+{ }^{t} G(\eta) \mathbb{L}\{\mathscr{F}(\eta+\xi)-\mathscr{F} \eta\}
\end{aligned}
$$

As shown in $[8]$, the Fréchet derivative $\Phi_{\eta}(t ; \mathscr{F} \eta)$ of the matrizant $\Phi(t ; \mathscr{F} \eta)$ with respect to $\eta$ exists and is represented by

$$
\begin{aligned}
& \Phi_{\eta}(t ; \mathscr{F} \eta) \xi \\
& \quad=\Phi(t ; \mathscr{F} \eta) \int_{a}^{t} \Phi^{-1}(s ; \mathscr{F} \eta) X_{x x}[s, \mathscr{F} \eta(s)] \Phi(s ; \mathscr{F} \eta) \xi \Phi(s ; \mathscr{F} \eta) d s .
\end{aligned}
$$

Hence the equation

$$
\left.\mathscr{T}_{\eta}(\eta, 0)={ }^{t}\left(\mathbb{L} \Phi_{\eta}(\cdot ; \mathscr{F} \eta) \xi\right)\{\mathbb{L} \mathscr{F} \eta-d\}\right\}+\mathscr{b}(\eta) \xi
$$

holds.

Let us consider $\lim _{\varepsilon \rightarrow 0} \mathscr{T}_{\eta}(\eta, \varepsilon)$ in (2.22). By (1.20) and (1.23) we have

$$
u_{2}^{(j)}(t)=\frac{1}{\varepsilon}\left\{\mathscr{F}\left(\eta+\varepsilon e_{j}\right)(t)-\mathscr{F} \eta(t)\right\}=\varphi^{(j)}(t)+v^{(j)}(t)
$$

and

$$
\left\|v^{(j)}\right\|_{C} \leqq C^{*}|\varepsilon|,
$$

hence $u_{2}^{(j)} \rightarrow \varphi^{(j)}$ as $\varepsilon \rightarrow 0$. Moreover, as $\varepsilon \rightarrow 0$ the matrizant $\Psi_{j}(t ; \mathscr{F} \eta, \varepsilon)$, which corresponds to

$$
X_{x}[t, \mathscr{F} \eta(t)]+\varepsilon X_{x x}[t, \mathscr{F} \eta(t)] u_{2}^{(j)}(t),
$$

has the limit $\Psi_{j}(t ; \mathscr{F} \eta, 0)$ which is the matrizant corresponding to $X_{x}[t, \mathscr{F} \eta(t)]$. That is, $\Psi_{j}(t ; \mathscr{F} \eta, 0)$ is identical to $\Phi(t ; \mathscr{F} \eta)$ for every $j$. Therefore

$$
\begin{aligned}
& {\left[H_{j, \varepsilon} Y \Phi(\cdot ; \mathscr{F} \eta) \xi u_{2}^{(j)}\right](t)} \\
& \quad=\Psi_{j}(t ; \mathscr{F} \eta, \varepsilon) \int_{a}^{t} \Psi_{j}^{-1}(s ; \mathscr{F} \eta, \varepsilon) X_{x x}[s, \mathscr{F} \eta(s)] \Phi(s ; \mathscr{F} \eta) \xi u_{2}^{(j)}(s) d s \\
& \quad \rightarrow \Phi(t ; \mathscr{F} \eta) \int_{a}^{t} \Phi^{-1}(s ; \mathscr{F} \eta) X_{x x}[s, \mathscr{F} \eta(s)] \Phi(s ; \mathscr{F} \eta) \xi \varphi^{(j)}(s) d s
\end{aligned}
$$

holds as $\varepsilon \rightarrow 0$. In such limitation,

$$
\varepsilon h_{1}^{(j)}(t) \xi+\varepsilon^{2} h_{2}^{(j)}(t) \xi \rightarrow 0,
$$

which implies

$$
P(t ; \mathscr{F} \eta, \varepsilon) \xi \rightarrow \Phi_{\eta}(t ; \mathscr{F} \eta) \xi
$$


as $\varepsilon \rightarrow 0$. Hence, we obtain the equation

$$
\begin{aligned}
\lim _{\varepsilon \rightarrow 0} \mathscr{T}_{\eta}(\eta, \varepsilon) \xi & ={ }^{t}\left(\boldsymbol{L} \Phi_{\eta}(\cdot ; \mathscr{F} \eta) \xi\right)\{\boldsymbol{L} \mathscr{F} \eta-\boldsymbol{d}\}+\mathscr{b}(\eta) \xi \\
& =\mathscr{T}_{\eta}(\eta, 0) \xi,
\end{aligned}
$$

which implies the continuity of $\mathscr{T}_{\eta}(\eta, \varepsilon)$ at $\varepsilon=0$.

Third stage. For the isolated minimal point $\eta^{*}$, we have

$$
\mathscr{T}_{\eta}\left(\eta^{*}, 0\right) \xi=\mathfrak{G}\left(\eta^{*}\right) \xi+{ }^{t}\left(\boldsymbol{L} \Phi_{\eta}\left(\cdot ; \mathscr{F} \eta^{*}\right) \xi\right)\left\{\boldsymbol{L} \mathscr{F} \eta^{*}-\boldsymbol{d}\right\} .
$$

Then,

$$
\begin{aligned}
{ }^{t}( & \left.\boldsymbol{L} \Phi_{\eta}\left(\cdot ; \mathscr{F} \eta^{*}\right) \xi\right)\left\{\boldsymbol{L} \mathscr{F} \eta^{*}-\boldsymbol{d}\right\} \\
={ }^{t} & \left(\boldsymbol{L} \Phi\left(\cdot ; \mathscr{F} \eta^{*}\right) \int_{a} \Phi^{-1}\left(s ; \mathscr{F} \eta^{*}\right) X_{x x}\left[s, \mathscr{F} \eta^{*}(s)\right] \Phi\left(s ; \mathscr{F} \eta^{*}\right) \xi \Phi\left(s ; \mathscr{F} \eta^{*}\right) d s\right) \\
& \times\left\{\boldsymbol{L} \mathscr{F} \eta^{*}-\boldsymbol{d}\right\} \\
= & { }^{t}\left(p_{N} \int_{a}^{\cdot} \Phi^{-1}\left(s ; \mathscr{F} \eta^{*}\right) X_{x x}\left[s, \mathscr{F} \eta^{*}(s)\right] \Phi\left(s ; \mathscr{F} \eta^{*}\right) \xi \Phi\left(s ; \mathscr{F} \eta^{*}\right) d s\right) \\
& \quad \times{ }^{t} G\left(\eta^{*}\right)\left\{\boldsymbol{L} \mathscr{F} \eta^{*}-\boldsymbol{d}\right\},
\end{aligned}
$$

where $p_{N}$ means an operation such that $p_{N} y={ }^{t}\left(y\left(t_{1}\right), \ldots, y\left(t_{N}\right)\right)$ for $y \in C(I)$. Because of (1.13) in Assumption 2, the above term is equal to 0. Hence, from (2.26) the relation

$$
\mathscr{T}_{\eta}\left(\eta^{*}, 0\right) \xi=\mathfrak{G}\left(\eta^{*}\right) \xi
$$

holds, which implies the invertibility of $\mathscr{T}_{\eta}\left(\eta^{*}, 0\right)$ by virtue of $(1.14)$ in Assumption 2.

By the implicit function theorem (Th. 1.7 in [3]), we have the desired conclusion.

\section{§3. Convergence of the Iterative Process}

In the preceding section we have shown the existence of a fixed point for the operator $\mathscr{A}$ in $N b$. We shall denote the fixed point by $\hat{\eta}=\hat{\eta}(\varepsilon)$ and call it the approximate minimal point of $J(\eta)$ because it is an approximation of $\eta^{*}$ and tends to $\eta^{*}$ as $\varepsilon \rightarrow 0$.

In this section we shall show that in some neighbourhood of $\hat{\eta}$ the iterative process (1.19) converges to $\hat{\eta}$ while $\varepsilon$ is fixed as a positive number less than $\varepsilon_{1}$.

Since $\hat{\eta}$ is defined by $\mathscr{T}(\hat{\eta}, \varepsilon)=0$, the equation

$$
\left\{{ }^{t} S(\varepsilon ; \mathscr{F} \hat{\eta}) S(\varepsilon ; \mathscr{F} \hat{\eta})\right\}^{-1}{ }^{t} S(\varepsilon ; \mathscr{F} \hat{\eta})\{\boldsymbol{L} \mathscr{F} \hat{\eta}-\boldsymbol{d}\}=0
$$


holds as far as $\left\|\hat{\eta}-\eta^{*}\right\| \leqq \Delta$.

On the convergence we have the following theorem.

Theorem 2. There exists a positive number $\Delta_{2}$ such that the iterative process (1.19) starting with any $\eta_{0}=\eta$ in the $\Delta_{2}$-neighbourhood of $\hat{\eta}$ converges to $\hat{\eta}$.

Proof. Let $\Delta_{2}$ be sufficiently small such that the $\Delta_{2}$-neighbourhood of $\hat{\eta}$ is included in $B_{\Delta}$. We shall show that $\mathscr{A}$ is a contraction mapping on the $\Delta_{2}$ neighbourhood of $\hat{\eta}$.

We have

$$
\begin{aligned}
\mathscr{A} \eta-\hat{\eta}= & \eta-\hat{\eta}-\left\{{ }^{t} S(\varepsilon ; \mathscr{F} \eta) S(\varepsilon ; \mathscr{F} \eta)\right\}^{-1} \mathscr{T}(\eta, \varepsilon) \\
= & \eta-\hat{\eta}-\left\{{ }^{t} S(\varepsilon ; \mathscr{F} \eta) S(\varepsilon ; \mathscr{F} \eta)\right\}^{-1}\{\mathscr{T}(\eta, \varepsilon)-\mathscr{T}(\hat{\eta}, \varepsilon)\} \\
= & \left\{{ }^{t} S(\varepsilon ; \mathscr{F} \eta) S(\varepsilon ; \mathscr{F} \eta)\right\}^{-1}\left\{{ }^{t} S(\varepsilon ; \mathscr{F} \eta) S(\varepsilon ; \mathscr{F} \eta)(\eta-\hat{\eta})\right. \\
& \left.-\mathscr{T}_{\eta}(\hat{\eta}, \varepsilon)(\eta-\hat{\eta})\right\}+o(\|\eta-\hat{\eta}\|) .
\end{aligned}
$$

Because of (2.22), the equation

$$
\begin{aligned}
& { }^{t} S(\varepsilon ; \mathscr{F} \eta) S(\varepsilon ; \mathscr{F} \eta)(\eta-\hat{\eta})-\mathscr{T}_{\eta}(\hat{\eta}, \varepsilon)(\eta-\hat{\eta}) \\
& ={ }^{t} S(\varepsilon ; \mathscr{F} \eta) S(\varepsilon ; \mathscr{F} \eta)(\eta-\hat{\eta})-{ }^{t} S(\varepsilon ; \mathscr{F} \hat{\eta}) G(\hat{\eta})(\eta-\hat{\eta}) \\
& \quad-{ }^{t}\{\boldsymbol{L} P(\cdot ; \mathscr{F} \hat{\eta}, \varepsilon)(\eta-\hat{\eta})\}\{\boldsymbol{L} \mathscr{F} \hat{\eta}-\boldsymbol{d}\}+o(\|\eta-\hat{\eta}\|)
\end{aligned}
$$

holds. The first term of the right-hand side of the above equation is written by

$$
\begin{aligned}
\left\{{ }^{t} S(\varepsilon ; \mathscr{F} \eta) S(\varepsilon ; \mathscr{F} \eta)-{ }^{t} S(\varepsilon ; \mathscr{F} \hat{\eta}) G(\hat{\eta})\right\}(\eta-\hat{\eta}) \\
=\left\{{ }^{t} S(\varepsilon ; \mathscr{F} \eta) S(\varepsilon ; \mathscr{F} \eta)-{ }^{t} S(\varepsilon ; \mathscr{F} \hat{\eta}) S(\varepsilon ; \mathscr{F} \hat{\eta})\right\}(\eta-\hat{\eta}) \\
\quad+{ }^{t} S(\varepsilon ; \mathscr{F} \hat{\eta})\{S(\varepsilon ; \mathscr{F} \hat{\eta})-G(\hat{\eta})\}(\eta-\hat{\eta}) .
\end{aligned}
$$

By virtue of (2.12), the estimation for the compound adjusting matrices

$$
\left\|{ }^{t} S(\varepsilon ; \mathscr{F} \eta) S(\varepsilon ; \mathscr{F} \eta)-{ }^{t} S(\varepsilon ; \mathscr{F} \hat{\eta}) S(\varepsilon ; \mathscr{F} \hat{\eta})\right\| \leqq \text { const. }\|\eta-\hat{\eta}\|
$$

holds, which brings

$$
\left\{{ }^{t} S(\varepsilon ; \mathscr{F} \eta) S(\varepsilon ; \mathscr{F} \eta)-{ }^{t} S(\varepsilon ; \mathscr{F} \hat{\eta}) S(\varepsilon ; \mathscr{F} \hat{\eta})\right\}(\eta-\hat{\eta})=o(\|\eta-\hat{\eta}\|) .
$$

Utilizing the inequality (1.24), we have

$$
\left\|{ }^{t} S(\varepsilon ; \mathscr{F} \hat{\eta})\{S(\varepsilon ; \mathscr{F} \hat{\eta})-G(\hat{\eta})\}\right\| \leqq\left(L_{0} M_{0}+\delta_{1}\right) \delta_{1} .
$$

On the other hand, the principal part of the second term of (3.3) has the following $j$-th column vector

$$
{ }^{t}\left(\mathbb{L} \Psi_{j}(\cdot ; \mathscr{F} \hat{\eta}, \varepsilon) \int_{a}^{\cdot} \Psi_{j}^{-1}(s ; \mathscr{F} \hat{\eta}, \varepsilon) X_{x x}[s, \mathscr{F} \hat{\eta}(s)] \Phi(s ; \mathscr{F} \hat{\eta})(\eta-\hat{\eta}) u_{2}^{(j)}(s) d s\right)
$$




$$
\begin{aligned}
& \times\{\boldsymbol{L} \mathscr{F} \hat{\eta}-\boldsymbol{d}\} \\
= & { }^{t}\left(p_{N} \int_{a}^{\cdot} \Psi_{j}^{-1}(s ; \mathscr{F} \hat{\eta}, \varepsilon) X_{x x}[s, \mathscr{F} \hat{\eta}(s)] \Phi(s ; \mathscr{F} \hat{\eta})(\eta-\hat{\eta}) u_{2}^{(j)}(s) d s\right) \\
& \times{ }^{t}\left(\boldsymbol{L} \Psi_{j}(\cdot ; \mathscr{F} \hat{\eta}, \varepsilon)\right)\{\boldsymbol{L} \mathscr{F} \hat{\eta}-\boldsymbol{d}\},
\end{aligned}
$$

where $p_{N}$ means the same operation in Section 2. Then we have the estimation

$$
\begin{aligned}
& \boldsymbol{L} \Psi_{j}(\cdot ; \mathscr{F} \hat{\eta}, \varepsilon)-S(\varepsilon ; \mathscr{F} \hat{\eta}) \\
& \quad=\boldsymbol{L} \Psi_{j}(\cdot ; \mathscr{F} \hat{\eta}, \varepsilon)-G(\hat{\eta})+G(\hat{\eta})-S(\varepsilon ; \mathscr{F} \hat{\eta}) \\
& \quad=o(\varepsilon)
\end{aligned}
$$

because of (1.24) and the same assertion in the proof of Theorem 1.

Therefore, we have

(the $j$-th column vector of $\left.{ }^{t}\{\boldsymbol{L} P(\cdot ; \mathscr{F} \hat{\eta}, \varepsilon)(\eta-\hat{\eta})\}\{\boldsymbol{L} \mathscr{F} \hat{\eta}-\boldsymbol{d}\}\right)$

$$
\begin{aligned}
= & { }^{t}\left(p_{N} \int_{a} \Psi_{j}^{-1}(s ; \mathscr{F} \hat{\eta}, \varepsilon) X_{x x}[s, \mathscr{F} \hat{\eta}(s)] \Phi(s ; \mathscr{F} \hat{\eta})(\eta-\hat{\eta}) u_{2}^{(j)}(s) d s\right) \\
& \times{ }^{t} S(\varepsilon ; \mathscr{F} \hat{\eta})\{\boldsymbol{L} \mathscr{F} \hat{\eta}-\boldsymbol{d}\}+o(\varepsilon)(\eta-\hat{\eta}),
\end{aligned}
$$

which implies the estimation

$$
\left\|^{t}\{\boldsymbol{L} P(\cdot ; \mathscr{F} \hat{\eta}, \varepsilon)(\eta-\hat{\eta})\}\{\boldsymbol{L} \mathscr{F} \hat{\eta}-\boldsymbol{d}\}\right\| \leqq o(\varepsilon)\|\eta-\hat{\eta}\|
$$

because of (3.1).

Remembering the estimation (1.25) and summing up (3.4), (3.5) and (3.6), we have

$$
\begin{aligned}
& \left\|\eta-\hat{\eta}-\left\{{ }^{t} S(\varepsilon ; \mathscr{F} \eta) S(\varepsilon ; \mathscr{F} \eta)\right\}^{-1}{ }^{t} S(\varepsilon ; \mathscr{F} \hat{\eta}) G(\hat{\eta})(\eta-\hat{\eta})\right\| \\
& \quad \leqq \frac{\delta_{1} M_{1}\left(L_{0} M_{0}+\delta_{1}\right)}{1-\delta_{1} M_{1}\left(2 L_{0} M_{0}+\delta_{1}\right)}\|\eta-\hat{\eta}\| .
\end{aligned}
$$

Thus choose $\varepsilon$ and $\|\eta-\hat{\eta}\|$ sufficiently small such that the inequality

$$
\|\mathscr{A} \eta-\hat{\eta}\| \leqq\left(\frac{\delta_{1} M_{1}\left(L_{0} M_{0}+\delta_{1}\right)}{1-\delta_{1} M_{1}\left(2 L_{0} M_{0}+\delta_{1}\right)}+\alpha\right)\|\eta-\hat{\eta}\|
$$

holds, where $\alpha$ is a positive number satisfying the inequality

$$
\alpha \leqq \frac{1-\delta_{1} L_{0} M_{0} M_{1}}{1-\delta_{1} M_{1}\left(2 L_{0} M_{0}+\delta_{1}\right)}(1-\kappa)
$$

for an arbitrary positive $\kappa<1$. Therefore, we have

$$
\|\mathscr{A} \eta-\hat{\eta}\| \leqq(1-\kappa)\|\eta-\hat{\eta}\|,
$$

which shows the contraction property of $\mathscr{A}$.

From the above considerations we have the assertion as in [5] that the 
convergence of the iteration would not be expected to be quadratic in the neighbourhood of $\hat{\eta}$ while a fixed $\varepsilon$ is chosen to be apart from zero. The reason is the following. The equation

$$
\begin{aligned}
& \mathscr{A}(\hat{\eta}+\xi)-\mathscr{A} \hat{\eta} \\
& \quad=\mathscr{A}(\hat{\eta}+\xi)-\hat{\eta} \\
& \quad=\xi-\left\{{ }^{t} S(\varepsilon ; \mathscr{F}(\hat{\eta}+\xi)) S(\varepsilon ; \mathscr{F}(\hat{\eta}+\xi))\right\}^{-1}\{\mathscr{T}(\hat{\eta}+\xi, \varepsilon)-\mathscr{T}(\hat{\eta}, \varepsilon)\},
\end{aligned}
$$

implies that the Fréchet derivative of $\mathscr{A}$ at $\hat{\eta}$ is given by

$$
\begin{aligned}
\mathscr{A}^{\prime}(\hat{\eta}) \xi= & \breve{\zeta}-\left\{{ }^{t} S(\varepsilon ; \mathscr{F} \hat{\eta}) S(\varepsilon ; \mathscr{F} \hat{\eta})\right\}^{-1}\left[{ }^{t}(\mathbb{L} P(\cdot ; \mathscr{F} \hat{\eta}, \varepsilon) \xi)\{\mathbb{L} \mathscr{F} \hat{\eta}-\boldsymbol{d}\}\right. \\
& \left.+{ }^{t} S(\varepsilon ; \mathscr{F} \hat{\eta}) G(\hat{\eta}) \xi\right] \\
= & \left\{{ }^{t} S(\varepsilon ; \mathscr{F} \hat{\eta}) S(\varepsilon ; \mathscr{F} \hat{\eta})\right\}^{-1}\left[{ }^{t} S(\varepsilon ; \mathscr{F} \hat{\eta})\{S(\varepsilon ; \mathscr{F} \hat{\eta})-G(\hat{\eta})\} \xi\right. \\
& \left.-{ }^{t}(\boldsymbol{L} P(\cdot ; \mathscr{F} \hat{\eta}, \varepsilon) \xi)\{\boldsymbol{L} \mathscr{F} \hat{\eta}-\boldsymbol{d}\}\right] .
\end{aligned}
$$

As in the derivation of (3.7), we have

$$
{ }^{t}(\boldsymbol{L P}(\cdot ; \mathscr{F} \hat{\eta}, \varepsilon) \xi)\{\boldsymbol{L} \mathscr{F} \hat{\eta}-\boldsymbol{d}\}=o(\varepsilon) \xi .
$$

On the other hand, $S(\varepsilon ; \mathscr{F} \hat{\eta})-G(\hat{\eta})$ does not vanish if $\varepsilon \neq 0$. Then, we can not expect that $\mathscr{A}^{\prime}(\hat{\eta}) \xi$ vanishes for any $\xi \neq 0$. Hence the convergence is not quadratical.

If we assume that $X_{x x}[t, x]$ is identically zero on $\mathscr{D}$ (almost linear case for differential equation), then the convergence is quadratic because in such case $v^{(j)}(t)$ in $(1.20)$ is identically zero and $S(\varepsilon ; \mathscr{F} \eta)=G(\eta)$ holds, which makes our iterative process very simple and guarantees the quadratic convergence.

Remark. In [8], Urabe mentioned the convergence rate of the several iterative processes, and wrote that the quasilinearization algorithm by Banks and Groome is not quadratically convergent because it is not the Newton iterative process (p. 182 of [8]). But, the sufficient condition of the quadratic convergence is the zero spectral radius of the Fréchet derivative of the nonlinear operator representing the iterative process at the limit point (fixed point of the operator), and the quasilinearization algorithm has such property as shown in [1].

Concluding this section, we investigate the approximation order of $\hat{\eta}$ for $\eta^{*}$. We shall improve the statement of Lemma 2.

Lemma 4. For $\eta \in B_{\Delta}$ and $|\varepsilon|,|\varepsilon+v| \leqq \varepsilon_{0}$, the equation

$$
S(\varepsilon+v ; \mathscr{F} \eta)-S(\varepsilon ; \mathscr{F} \eta)=\mathbb{L} p(\cdot ; \mathscr{F} \eta, \varepsilon) v+o(|v|)
$$


holds, where $p(t ; \mathscr{F} \eta, \varepsilon)$ is an $n \times n$ matrix with components of continuous functions on $I$.

Proof. After the notations in Lemma 2, let us put

$$
\begin{aligned}
& \mathscr{F}\left(\eta+(\varepsilon+v) e_{j}\right)=y_{1}^{(j)}=x+(\varepsilon+v)\left\{\varphi^{(j)}+v_{1}^{(j)}\right\}, \\
& \mathscr{F}\left(\eta+\varepsilon e_{j}\right)=y_{2}^{(j)}=x+\varepsilon\left\{\varphi^{(j)}+v_{2}^{(j)}\right\}
\end{aligned}
$$

and

$$
w^{(j)}=v_{1}^{(j)}-v_{2}^{(j)}
$$

Then we have

$$
s_{j}(\varepsilon+v ; \mathscr{F} \eta)-s_{j}(\varepsilon ; \mathscr{F} \eta)=\boldsymbol{L} w^{(j)},
$$

and $w^{(j)}$ must satisfy the differential equation

$$
\begin{aligned}
\frac{d w^{(j)}}{d t}= & \frac{1}{\varepsilon+v}\left\{X\left[t, y_{1}^{(j)}(t)\right]-X[t, x(t)]\right\} \\
& -\frac{1}{\varepsilon}\left\{X\left[t, y_{2}^{(j)}(t)\right]-X[t, x(t)]\right\}, \quad a<t<b
\end{aligned}
$$

and the initial condition

$$
w^{(j)}(a)=0
$$

just as (2.6) and (2.7). Utilizing the equations (1.7) and (1.8), we have (the right-hand side of (3.10))

$$
\begin{aligned}
= & \frac{1}{\varepsilon+v}\left\{X_{x}[t, x(t)](\varepsilon+v)\left(\varphi^{(j)}+v_{1}^{(j)}\right)\right. \\
& +\frac{1}{2} X_{x x}[t, x(t)](\varepsilon+v)^{2}\left(\varphi^{(j)}+v_{1}^{(j)}\right)\left(\varphi^{(j)}+v_{1}^{(j)}\right) \\
& \left.+Q\left[(\varepsilon+v)\left(\varphi^{(j)}+v_{1}^{(j)}\right) ; x\right]\right\}-\frac{1}{\varepsilon}\left\{X_{x}[t, x(t)] \varepsilon\left(\varphi^{(j)}+v_{2}^{(j)}\right)\right. \\
& \left.+\frac{1}{2} X_{x x}[t, x(t)] \varepsilon^{2}\left(\varphi^{(j)}+v_{2}^{(j)}\right)\left(\varphi^{(j)}+v_{2}^{(j)}\right)+Q\left[\varepsilon\left(\varphi^{(j)}+v_{2}^{(j)}\right) ; x\right]\right\} \\
= & X_{x}[t, x(t)] w^{(j)}+\frac{1}{2}(\varepsilon+v) X_{x x}[t, x(t)]\left(\varphi^{(j)}+v_{1}^{(j)}\right)\left(\varphi^{(j)}+v_{1}^{(j)}\right) \\
& -\frac{1}{2} \varepsilon X_{x x}[t, x(t)]\left(\varphi^{(j)}+v_{2}^{(j)}\right)\left(\varphi^{(j)}+v_{2}^{(j)}\right)+(\varepsilon+v)^{2} Q\left(\varphi^{(j)}+v_{1}^{(j)} ; x\right) \\
& -\varepsilon^{2} Q\left(\varphi^{(j)}+v_{2}^{(j)} ; x\right) \\
= & \left\{X_{x}[t, x(t)]+\varepsilon X_{x x}[t, x(t)]\left(\varphi^{(j)}+v_{2}^{(j)}\right)+\varepsilon^{2} q_{2}\left(\varphi^{(j)}+v_{2}^{(j)}\right)\right\} w^{(j)} \\
& +\frac{1}{2} v X_{x x}[t, x(t)]\left(\varphi^{(j)}+v_{2}^{(j)}\right)\left(\varphi^{(j)}+v_{2}^{(j)}\right)+2 v \varepsilon Q\left(\varphi^{(j)}+v_{2}^{(j)} ; x\right) \\
& +v X_{x x}[t, x(t)]\left(\varphi^{(j)}+v_{2}^{(j)}\right) w^{(j)}+\frac{1}{2}(\varepsilon+v) X_{x x}[t, x(t)] w^{(j)} w^{(j)} \\
& +\varepsilon^{2}\left\{\left(\varphi^{(j)}+v_{2}^{(j)}\right) q_{1}\left(w^{(j)}\right)+w^{(j)} q_{3}\left(w^{(j)}\right)\right\},
\end{aligned}
$$


where $q_{3}\left(w^{(j)}, 0\right)$ is abbreviated by $q_{3}\left(w^{(j)}\right)$.

Let $\Omega_{j}(t ; \mathscr{F} \eta, \varepsilon)$ be the matrizant which corresponds to

$$
X_{x}[t, x(t)]+\varepsilon X_{x x}[t, x(t)]\left(\varphi^{(j)}+v_{2}^{(j)}\right)+\varepsilon^{2} q_{2}\left(\varphi^{(j)}+v_{2}^{(j)}\right)
$$

and transform (3.10) and (3.11) into the integral equation

$$
\begin{aligned}
w^{(j)}(t)= & \nu \Omega_{j}(t ; \mathscr{F} \eta, \varepsilon) \int_{a}^{t} \Omega_{j}^{-1}(s ; \mathscr{F} \eta, \varepsilon) \Xi_{j}(s ; \mathscr{F} \eta, \varepsilon) d s \\
& +\Omega_{j}(t ; \mathscr{F} \eta, \varepsilon) \int_{a}^{t} \Omega_{j}^{-1}(s ; \mathscr{F} \eta, \varepsilon) W_{j}\left(s ; w^{(j)}\right) d s .
\end{aligned}
$$

Here

$$
\begin{aligned}
\Xi_{j}(t ; \mathscr{F} \eta, \varepsilon)= & \frac{1}{2} X_{x x}[t, x(t)]\left\{\varphi^{(j)}(t)+v_{2}^{(j)}(t)\right\}\left\{\varphi^{(j)}(t)+v_{2}^{(j)}(t)\right\} \\
& +2 \varepsilon Q\left(\varphi^{(j)}(t)+v_{2}^{(j)}(t) ; x\right)
\end{aligned}
$$

and

$$
\begin{aligned}
W_{j}\left(t ; w^{(j)}\right)= & v X_{x x}[t, x(t)]\left\{\varphi^{(j)}(t)+v_{2}^{(j)}(t)\right\} w^{(j)} \\
& +\frac{1}{2}(\varepsilon+v) X_{x x}[t, x(t)] w^{(j)} w^{(j)} \\
& +\varepsilon^{2}\left\{\left(\varphi^{(j)}(t)+v_{2}^{(j)}(t)\right) q_{1}\left(w^{(j)}\right)+w^{(j)} q_{3}\left(w^{(j)}\right)\right\} .
\end{aligned}
$$

Then, the first term of (3.12) is the order of $O(|v|)$ and the second term has no factors of the order lower than $O(|v|)$. Hence $w^{(j)}(t)$ is the order of $O(|v|)$ as a whole. Considering this fact, each term of $W_{j}$ is the order of $O\left(|v|^{2}\right)$.

Therefore we have the equation

$$
w^{(j)}(t)=v \Omega_{j}(t ; \mathscr{F} \eta, \varepsilon) \int_{a}^{t} \Omega_{j}^{-1}(s ; \mathscr{F} \eta, \varepsilon) \Xi_{j}(s ; \mathscr{F} \eta, \varepsilon) d s+o(|v|) .
$$

Put $p(t ; \mathscr{F} \eta, \varepsilon)$ such that $p$ has the $j$-th column vector equal to $w^{(j)} / v$, then we have (3.9).

Corollary. For $\eta \in B_{\Delta}$ and $|v| \leqq \varepsilon_{0}$, the equation

$$
S(v ; \mathscr{F} \eta)-G(\eta)=\boldsymbol{L} p(\cdot ; \mathscr{F} \eta) v+o(|v|)
$$

holds. Here $p(t ; \mathscr{F} \eta)$ is formed by

$$
\frac{1}{2} \Phi(t ; \mathscr{F} \eta) \int_{a}^{t} \Phi^{-1}(s ; \mathscr{F} \eta) X_{x x}[\mathscr{F} \eta(s), s] \varphi^{(j)}(s) \varphi^{(j)}(s) d s
$$

as the j-th column vector.

By virtue of Lemma 4 and its Corollary we obtain informations about the derivatives of $\mathscr{T}(\eta, \varepsilon)$ with respect to $\varepsilon$ at some neighbourhood of $\left(\eta^{*}, 0\right)$, which 
implies the following

Theorem 3. In the neighbourhood

$$
N b\left(\Delta_{3}, \varepsilon_{3}\right)=\left\{(\eta, \varepsilon) ;\left\|\eta-\eta^{*}\right\| \leqq \Delta_{3} \text { and }|\varepsilon| \leqq \varepsilon_{3}\right\},
$$

$\hat{\eta}(\varepsilon)$ whose existence is guaranteed in Theorem 1 has the estimation such as

$$
\left\|\hat{\eta}(\varepsilon)-\eta^{*}\right\| \leqq \text { const. }|\varepsilon| \text { as } \varepsilon \rightarrow 0
$$

Proof. We have shown in the third stage of the proof of Theorem 1 that $\mathscr{T}_{\eta}\left(\eta^{*}, 0\right)$ is identical to $\mathscr{S}\left(\eta^{*}\right)$ and is invertible. Taking the continuity of the derivative $\mathscr{T}_{\eta}(\eta, \varepsilon)$ at $\varepsilon=0$ into consideration, we can choose the numbers $\Delta_{3}$ and $\varepsilon_{3}$ such that $\mathscr{T}_{\eta}(\hat{\eta}, \varepsilon)$ has the bounded inverse in $N b\left(\Delta_{3}, \varepsilon_{3}\right)$.

On the other hand the equation

$$
\begin{aligned}
& \mathscr{T}(\hat{\eta}, \varepsilon+v)-\mathscr{T}(\hat{\eta}, \varepsilon) \\
& \quad={ }^{t} S(\varepsilon+v ; \mathscr{F} \hat{\eta})\{\boldsymbol{L} \mathscr{F} \hat{\eta}-\boldsymbol{d}\}-{ }^{t} S(\varepsilon ; \mathscr{F} \hat{\eta})\{\boldsymbol{L} \mathscr{F} \hat{\eta}-\boldsymbol{d}\} \\
& \quad={ }^{t}\{S(\varepsilon+v ; \mathscr{F} \hat{\eta})-S(\varepsilon ; \mathscr{F} \hat{\eta})\}\{\boldsymbol{L} \mathscr{F} \hat{\eta}-\boldsymbol{d}\}
\end{aligned}
$$

holds, and by Lemma 4 we obtain the assertion that $\mathscr{T}(\eta, \varepsilon)$ has the derivative with respect to $\varepsilon$ at $(\hat{\eta}(\varepsilon), \varepsilon)$ such as

$$
\mathscr{T}_{\varepsilon}(\hat{\eta}, \varepsilon) \nu={ }^{t}\{\boldsymbol{L} p(\cdot ; \mathscr{F} \hat{\eta}, \varepsilon) v\}\{\boldsymbol{L} \mathscr{F} \hat{\eta}-\boldsymbol{d}\} .
$$

Then, replacing $\Delta_{3}$ and $\varepsilon_{3}$ by suitable numbers less than them if necessary, Theorem 20.3 in [3] implies the existence of the derivatives of $\hat{\eta}(\varepsilon)$ in $N b\left(\Delta_{3}, \varepsilon_{3}\right)$, which is defined by

$$
\hat{\eta}_{\varepsilon}(\varepsilon) \nu=-\left(\mathscr{T}_{\eta}(\hat{\eta}, \varepsilon)\right)^{-1} \mathscr{T}_{\varepsilon}(\hat{\eta}, \varepsilon) v .
$$

Thus $\hat{\eta}(\varepsilon)$ is the order of $O(|\varepsilon|)$ as $\varepsilon \rightarrow 0$ in $N b\left(\Delta_{3}, \varepsilon_{3}\right)$.

Remark. Let $\varepsilon$ tend to 0 in (3.18), then we have

$$
\mathscr{T}_{\varepsilon}\left(\eta^{*}, 0\right) v={ }^{t}\left\{\boldsymbol{L} p\left(\cdot ; \mathscr{F} \eta^{*}\right) v\right\}\left\{\boldsymbol{L} \mathscr{F} \eta^{*}-\boldsymbol{d}\right\}
$$

by virtue of Corollary of Lemma 4 . By the fact

$$
\mathscr{T}_{\eta}\left(\eta^{*}, 0\right)=\mathfrak{G}\left(\eta^{*}\right)
$$

the equation

$$
\hat{\eta}_{\varepsilon}(0) v=-\mathfrak{S}^{-1}\left(\xi^{*}\right)^{t}\left\{\boldsymbol{L} p\left(\cdot ; \mathscr{F} \eta^{*}\right) v\right\}\left\{\boldsymbol{L} \mathscr{F} \eta^{*}-\boldsymbol{d}\right\}
$$

holds. However, we obtain

$$
{ }^{t}\left\{\boldsymbol{L} p\left(\cdot ; \mathscr{F} \eta^{*}\right) v\right\}
$$




$$
\begin{aligned}
& ={ }^{t}\left\{\frac{1}{2} v \boldsymbol{L} \Phi\left(\cdot ; \mathscr{F} \eta^{*}\right) \int_{a}^{*} \Phi^{-1}\left(s ; \mathscr{F} \eta^{*}\right) Z\left(s ; \mathscr{F} \eta^{*}\right) d s\right\} \\
& ={ }^{t}\left\{\frac{1}{2} v p_{N} \int_{a} \Phi^{-1}\left(s ; \mathscr{F} \eta^{*}\right) Z\left(s ; \mathscr{F} \eta^{*}\right) d s\right\}^{t} G\left(\eta^{*}\right),
\end{aligned}
$$

where $Z(t ; \mathscr{F} \eta)$ stands for the matrix which is composed by $X_{x x}[t, \mathscr{F} \eta(t)] \varphi^{(j)}(t)$ $\times \varphi^{(j)}(t)$ as the $j$-th column vector. Remembering that ${ }^{t} G\left(\eta^{*}\right)\left\{\boldsymbol{L} \mathscr{F} \eta^{*}-\mathbb{d}\right\}=0$, we obtain the statement that the derivative $\hat{\eta}_{\varepsilon}(0)$ vanishes, which implies that we can expect the approximate minimal point $\hat{\eta}(\varepsilon)$ to be the order of $o(|\varepsilon|)$ extremely near to $\eta^{*}$.

\section{§4. An Illustrative Example}

Let us consider the following problem originally mentioned in [4], which occurs relating to the tubular flow chemical reactor with axial mixing.

The differential equation is

$$
\frac{d^{2} x}{d \tau^{2}}-6 \frac{d x}{d \tau}-12 x^{2}=0, \quad 0<\tau<1 .
$$

By the transformation

$$
t=1-2 \tau,
$$

the equation (4.1) can be reduced to the following differential equation:

$$
\frac{d^{2} x}{d t^{2}}+3 \frac{d x}{d t}-3 x^{2}=0, \quad-1<t<1 .
$$

The constraining condition of least squares type is the following:

$$
\sum_{j=0}^{10}\left\{x\left(t_{j}\right)-y_{j}\right\}^{2}=\text { minimum }
$$

where

$$
t_{j}=0.2 j-1.0 \quad(j=0,1, \ldots, 10)
$$

and $y_{j}(j=0,1, \ldots, 10)$ are given in Table 4.1.

Table 4.1.

\begin{tabular}{ccccccc}
\hline \hline$j$ & 0 & 1 & 2 & 3 & 4 & 5 \\
$y_{j}$ & 0.38727 & 0.39476 & 0.41305 & 0.43862 & 0.47017 & 0.50764 \\
\hline & 6 & 7 & 8 & 9 & 10 & \\
& 0.55172 & 0.60372 & 0.66559 & 0.74012 & 0.83129 & \\
\hline
\end{tabular}


This problem is equivalent to the example in [2].

We shall rewrite the problem into the vector form as (1.1) and (1.2). The equation (4.3) is transformed into

$$
\left\{\begin{array}{l}
\frac{d x_{1}}{d t}=x_{2}, \\
\frac{d x_{2}}{d t}=3 x_{1}^{2}-3 x_{2},
\end{array}\right.
$$

Let $\boldsymbol{x}$ be the vector $\boldsymbol{x}=\left[\begin{array}{l}x_{1} \\ x_{2}\end{array}\right]$, then (4.6) is equivalent to

$$
\frac{d x}{d t}=\left[\begin{array}{l}
x_{2} \\
3 x_{1}^{2}-3 x_{2}
\end{array}\right], \quad-1<t<1
$$

and the functional value $J$ to be minimized is

$$
J=\frac{1}{2} \sum_{j=0}^{10}{ }_{t}\left[L_{j} x\left(t_{j}\right)-d_{j}\right]\left[L_{j} x\left(t_{j}\right)-d_{j}\right]
$$

Here $t_{j}(j=0,1, \ldots, 10)$ are the same as in $(4.5)$, the matrices $L_{j}(j=0,1, \ldots, 10)$ are

$$
L_{0}=L_{1}=\cdots=L_{10}=\left[\begin{array}{ll}
1 & 0 \\
0 & 0
\end{array}\right],
$$

and the vectors $d_{j}(j=0,1, \ldots, 10)$ are given by

$$
d_{j}=\left[\begin{array}{c}
y_{j} \\
0.0
\end{array}\right]
$$

The results of the numerical computation carried out by FACOM M-190 in the Data Processing Center, Kyoto University are shown in Table 4.2. For the numerical integration of ordinary differential equations the Runge-KuttaGill method programed by T. Ojika was used. All the calculations were carried out in the double precision arithmetic. 
Table 4.2.

\begin{tabular}{|c|c|c|c|c|}
\hline \multirow{2}{*}{ iteration times } & \multicolumn{2}{|c|}{0} & \multicolumn{2}{|c|}{1} \\
\hline & $x_{1}$ & $x_{2}$ & $x_{1}$ & $x_{2}$ \\
\hline$t_{0}=-1.0$ & 0.5 & 0.0 & 0.37806769956 & 0.10806510612 \\
\hline$t_{1}=-0.8$ & 0.51252090985 & 0.11507113359 & 0.40171204036 & 0.12821189883 \\
\hline$t_{2}=-0.6$ & 0.54334736769 & 0.18909213769 & 0.42937707807 & 0.14869156970 \\
\hline$t_{8}=-0.4$ & 0.58721750119 & 0.24863472740 & 0.46135283076 & 0.17162147360 \\
\hline$t_{4}=-0.2$ & 0.64281390289 & 0.30821629103 & 0.49830337268 & 0.19871943583 \\
\hline$t_{5}=0.0$ & 0.71115159268 & 0.37748477983 & 0.54123626991 & 0.23176626442 \\
\hline$t_{6}=0.2$ & 0.79503126629 & 0.46516837677 & 0.59155143668 & 0.27296261250 \\
\hline$t_{7}=$ & 0.89914650517 & 0.58192807415 & 0.65116185255 & 0.32531012072 \\
\hline$t_{8}=0.6$ & 1.0307690460 & 0.74350525298 & 0.72270288155 & 0.39313865121 \\
\hline$t_{9}=$ & 1.2012163950 & 0.97571861039 & 0.80987497209 & 0.48294851916 \\
\hline$t_{10}=$ & 1.4287209566 & 1.3241632086 & 0.91800946931 & 0.60486691312 \\
\hline$J_{k}$ & $4.93 \times 10^{-1}$ & & $1.16 \times 10^{-2}$ & \\
\hline \multirow{2}{*}{ iteration times } & \multicolumn{2}{|c|}{2} & \multicolumn{2}{|c|}{3} \\
\hline & $x_{1}$ & $x_{2}$ & $x_{1}$ & $x_{2}$ \\
\hline$t_{0}=-1.0$ & 0.38702086627 & 0.00261251861 & 0.38727177735 & -0.00004288586 \\
\hline$t_{1}=-0.8$ & 0.39490532424 & 0.07015010707 & 0.39476040385 & 0.06869739433 \\
\hline$t_{2}=-0.6$ & 0.41341909766 & 0.11229585764 & 0.41304637216 & 0.11139645273 \\
\hline$t_{3}=-0.4$ & 0.43913778607 & 0.14393243601 & 0.43861177501 & 0.14326111462 \\
\hline$t_{4}=-0.2$ & 0.47081948324 & 0.17287829974 & 0.47016701758 & 0.17226427328 \\
\hline$t_{5}=0.0$ & 0.50841638291 & 0.20369262002 & 0.50763820223 & 0.20303615170 \\
\hline$t_{6}=$ & 0.55263785706 & 0.23962085391 & 0.55171808024 & 0.23885031164 \\
\hline$t_{7}=$ & 0.60480645049 & 0.28369352839 & 0.60371555428 & 0.28274087195 \\
\hline$t_{8}=$ & 0.66689448238 & 0.33949736231 & 0.66558820885 & 0.33828049978 \\
\hline$t_{9}=$ & 0.74170948834 & 0.41193878825 & 0.74012428084 & 0.41034340656 \\
\hline$t_{10}=$ & 0.83325492973 & 0.50828488609 & 0.83129911340 & 0.50613875295 \\
\hline$J_{k}$ & $5.81 \times 10^{-6}$ & & $1.12 \times 10^{-10}$ & \\
\hline
\end{tabular}

\begin{tabular}{|c|c|c|}
\hline \multirow{2}{*}{ iteration times } & \multicolumn{2}{|r|}{4} \\
\hline & $x_{1}$ & $x_{2}$ \\
\hline$t_{0}=-1.0$ & 0.38727191330 & -0.00004431630 \\
\hline$t_{1}=-0.8$ & 0.39476032659 & 0.06869661205 \\
\hline$t_{2}=-0.6$ & 0.41304617227 & 0.11139596872 \\
\hline$t_{3}=-0.4$ & 0.43861149266 & 0.14326075367 \\
\hline$t_{4}=-0.2$ & 0.47016666726 & 0.17226394342 \\
\hline$t_{5}=0.0$ & 0.50763778440 & 0.20303579926 \\
\hline$t_{6}=$ & 0.55171758641 & 0.23884989812 \\
\hline$t_{7}=$ & 0.60371496863 & 0.28274036083 \\
\hline$t_{8}=$ & 0.66558750766 & 0.33827984706 \\
\hline$t_{9}=$ & 0.74012343005 & 0.41034255099 \\
\hline$t_{10}=$ & 0.83129806389 & 0.50613760232 \\
\hline$J_{k}$ & $1.10 \times 10^{-10}$ & \\
\hline
\end{tabular}

convergence criterion $1.0 \times 10^{-7}$

step size for the Runge-Kutta method 0.003125 $\varepsilon=1.0 \times 10^{-8}$ 


\section{References}

[1] Banks, H. T. and Groome, Jr., G. M., Convergence theorems for parameter estimation by quasilinearization, J. Math. Anal. Appl., 42 (1973), 91-109.

[2] Fujii, M., Numerical solutions to problems of the least squares type for ordinary differential equations by the use of Chebyshev series, Bull. Fukuoka Univ. Education, 27, Prat III (1978), 15-26.

[3] Красносельский, М. А., Вайникко, Г.М. и др., Приближенное решение операторных уравнений, "Наука", Москва, 1969.

[4] Lee, E. S., Quasilinearization and invariant imbedding, Academic P., New York, 1968.

[5] Mitsui, T., On the convergence of the initial-value adjusting method for nonlinear boundary value problems, Publ. RIMS, Kyoto Univ., 16 (1980), 513-529.

[6] Ojika, T. and Kasue, Y., Initial-value adjusting method for the solution of nonlinear multipoint boudary-value problems, J. Math. Anal. Appl., 69 (1979), 359-371.

[7] Urabe, M., The Newton method and its application to boundary value problems with nonlinear boundary conditions, Proc. U.S. -Japan Sem. Different. Funct. Eqs., Benjamin, New York, 1967, 383-410.

[8] Urabe, M., On the Newton method to solve problems of the least squares type for ordinary differential equations, Mem. Fac. Sci., Kyushu Univ., Ser. A, 29 (1975), 173-183. 